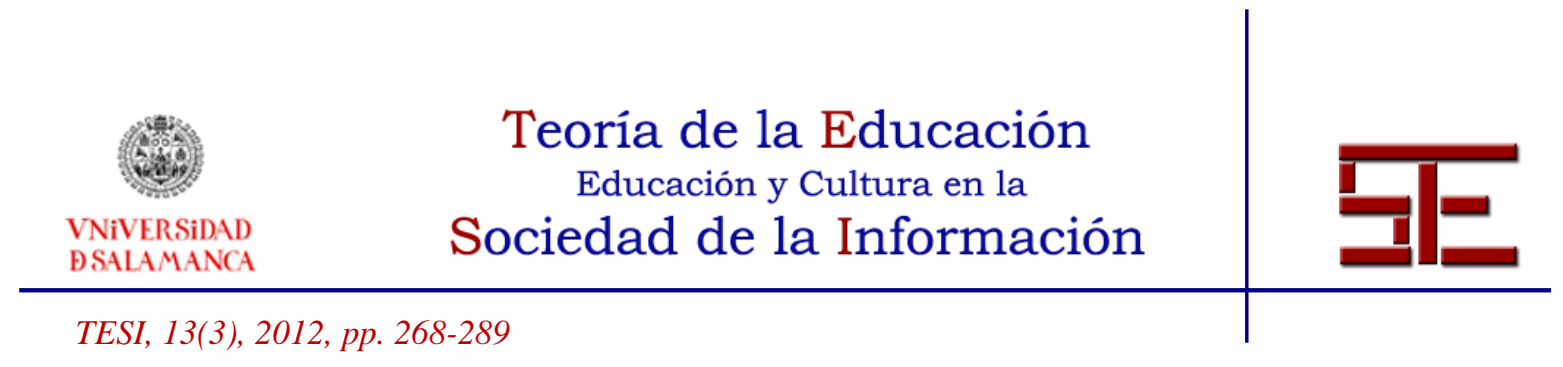

\title{
EVALUACIÓN DE LA FORMACIÓN CONTINUA EN EL SECTOR SANITARIO. RESULTADOS DE LA FORMACIÓN EN USO RACIONAL DEL MEDICAMENTO
}

Resumen: La evaluación de la eficacia de la formación es un reto para las organizaciones de hoy en día. En este artículo presentamos los resultados de una evaluación llevada a cabo para medir la transferencia de un plan de formación sobre el uso racional de medicamentos dirigido a 1550 profesionales del sector sanitario. Los datos se recogen mediante una encuesta al participante, dos meses después de la formación. Los resultados muestran que muchos de los participantes mejoraron su actuación profesional, en especial en sus funciones relacionadas con la selección y uso de medicamentos. También se detectan factores clave para la transferencia, tales como la motivación y el apoyo de la organización. En el artículo, reflexionamos sobre los puntos fuertes y débiles del plan de formación para así ayudar a otros investigadores/as y prácticos/as a avanzar en la mejora de la transferencia y la eficacia de la formación.

Palabras clave: Resultados de la formación; eficacia de la formación; transferencia de la formación; evaluación de la transferencia; sector sanitario.

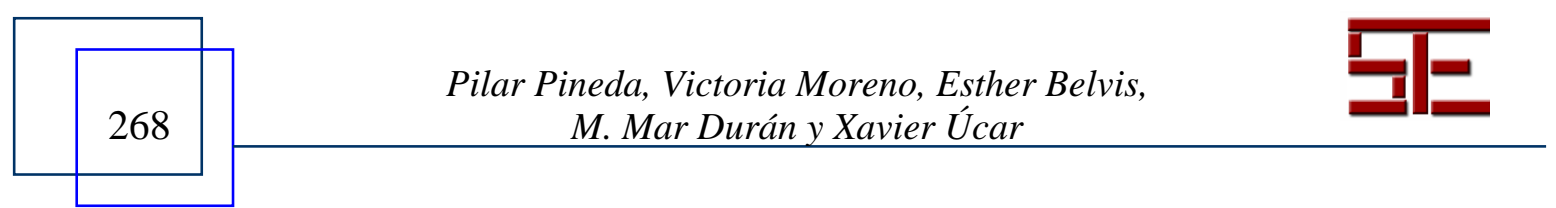




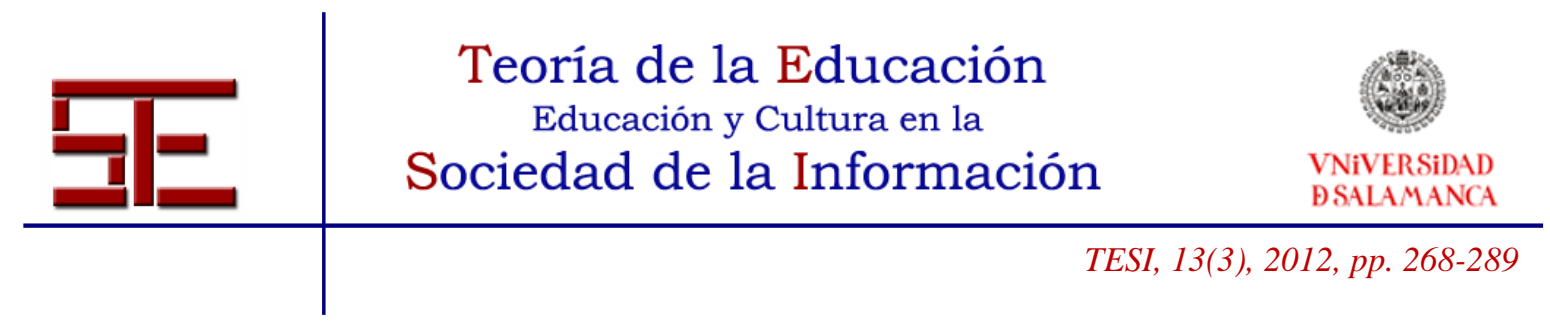

\title{
EVALUATION OF TRAINING IN THE HEALTH SECTOR. RESULTS OF TRAINING ON THE USE OF MEDICINES
}

\begin{abstract}
The evaluation of training efficacy is one of the challenges of today's organizations. In this article we present the results of an evaluation carried out in order to measure transfer of a training plan about the rational use of medicines addressed to 1550 health professionals. Data is collected by means of a survey to the trainee, two months after the training program. Results show that most of the trainees have improved their professional performance, in special the duties related to the selection and use of medicines. Key factors for transfer are also detected, such as motivation and support from the organization. In the paper, we reflect on the strengths and weaknesses of the training plan so as to help other researchers and practitioners to advance towards improving transfer of training and the efficacy of training.
\end{abstract}

Keywords: Training results; training effectiveness; transfer of training; evaluation of transfer; heath sector.

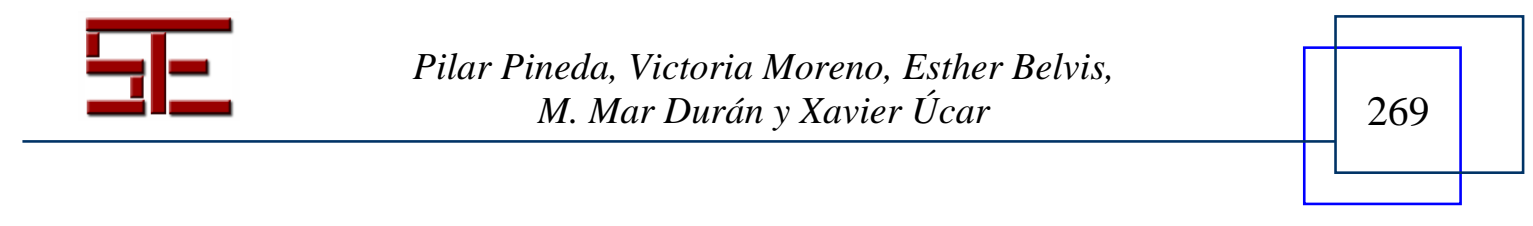




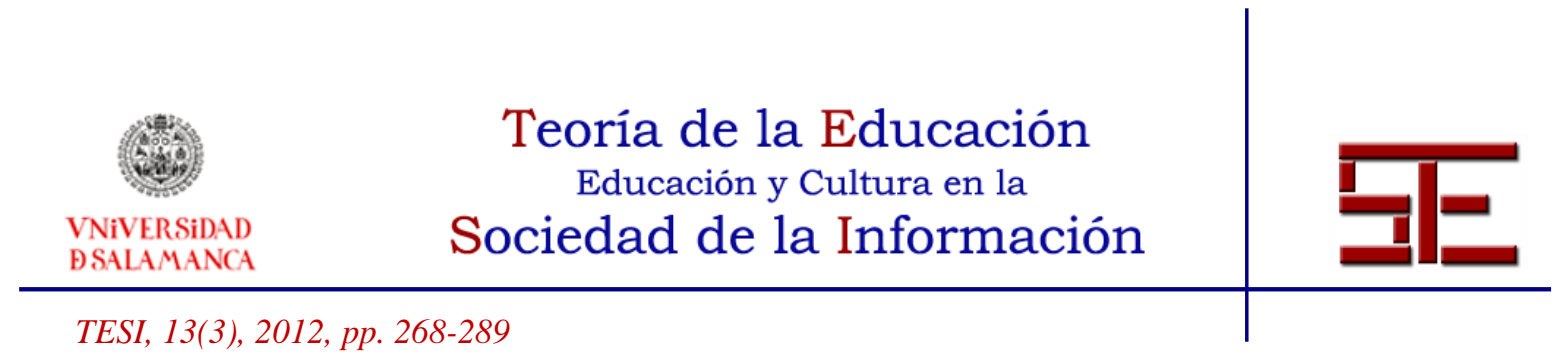

\section{EVALUACIÓN DE LA FORMACIÓN CONTINUA EN EL SECTOR SANITARIO. RESULTADOS DE LA FORMACIÓN EN USO RACIONAL DEL MEDICAMENTO ${ }^{1}$}

Fecha de recepción: 30/07/2010; fecha de aceptación: 20/07/2012; fecha de publicación: 30/11/2012

Pilar Pineda Herrero

pilar.pineda@uab.es

Universidad Autónoma de Barcelona

Esther Belvis Pons

ebelvis.pons@gmail.com

Universidad Autónoma de Barcelona

M. Mar Duran-Belloch

mariadelmar.duran@uab.cat

Universidad Autónoma de Barcelona

Xavier Úcar Martínez

xavier.ucar@uab.es

Universidad Autónoma de Barcelona

\section{INTRODUCCIÓN}

Reducir el gasto sanitario y aumentar la eficacia de las inversiones públicas en sanidad es una de las prioridades en la mayoría de países con un Estado del bienestar. El gasto en la sanidad pública se distribuye en diversos ámbitos; entre ellos el de los medicamentos. Mejorar las prácticas médicas en un uso racional del medicamento supone una utilización más adecuada de los fondos públicos, y la formación de los médicos en este sentido puede convertirse en la estrategia clave para conseguirlo.

Varios estudios realizados en diversos sectores muestran que la aplicación de los resultados de la formación al puesto de trabajo no es alta, y que buena parte de los

\footnotetext{
${ }^{1}$ Agradecemos a la entidad Unió Consorci Formació la financiación de este estudio, que nos ha permitido obtener los resultados que aquí se presentan.
}

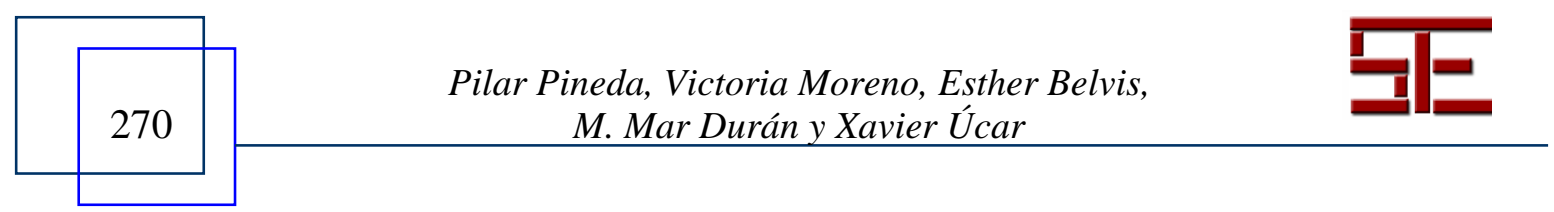




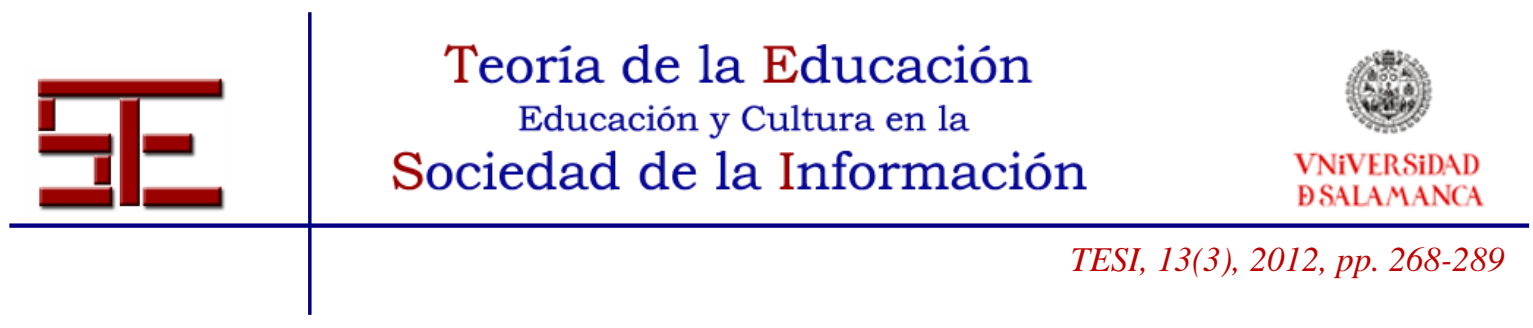

aprendizajes realizados no se llegan a aplicar, por lo que la formación pierde eficacia (Pineda y otros, 2007, 2008). Como indican Saks y Belcourt "Training professionals from 150 organizations reported that $62 \%, 44 \%$, and $34 \%$ of employees apply training material on the job immediately, six months, and one year after training. The average of these three values is 47\%" (Saks y Belcourt, 2006, 629). Transferir solamente el 50\% de lo aprendido al puesto de trabajo hace que la formación pierda, en cierta manera, su sentido y que pueda resultar incluso poco rentable desde el punto de vista organizacional. Esto exige la creación de modelos de transferencia, que posibiliten no solo evaluarla, sino sobre todo incrementarla para mejorar así la eficacia de la formación.

Diversos autores han elaborado modelos de evaluación de la formación,. Los más utilizados en la evaluación de la formación continua en nuestro contexto son el modelo de los cuatro niveles de Kirkpatrick (2005) y el modelo holístico de evaluación de Pineda (2002). Ambos plantean que la identificación de los resultados de la formación en términos de transferencia, es decir, la aplicación de los aprendizajes al puesto de trabajo, es el elemento central de la evaluación. Swanson (1996) en su modelo de evaluación PLS -performance, learning and satisfaction- insiste en la importancia de detectar las necesidades de mejora en la actuación profesional para poder evaluar los resultados de la formación en términos de aprendizaje y de cambios en el trabajo. Establece así un vínculo interesante entre necesidades-aprendizaje-resultados que resulta esencial para evaluar la formación. Su sistema de evaluación conecta los objetivos de actuación especificados en el análisis previo con los resultados de actuación obtenidos, por lo que su modelo resulta muy útil para evaluar la aplicación de la formación en el puesto de trabajo.

Otros autores han estudiado la transferencia de la formación, pero se han centrando más en el estudio de los factores que la determinan que en el desarrollo de modelos concretos para evaluar resultados. En este sentido destacan las aportaciones de Baldwin y Ford (1988), Thayer y Teachout (1995), Noe (1996), Holton (1996, 2005), Lim (2002), Nijman (2006) y Burke y Hutchins (2008), que permiten comprender los mecanismos que ayudan -o dificultan- la transferencia de la formación y elaborar así estrategias que la potencien e incluso instrumentos para diagnosticarla. Destaca el estudio realizado por Pidd (2004), en el que además de evaluar la transferencia de la formación de un programa de formación en el uso de drogas, se analizan factores clave que afectan a la transferencia como las características personales de los participantes y

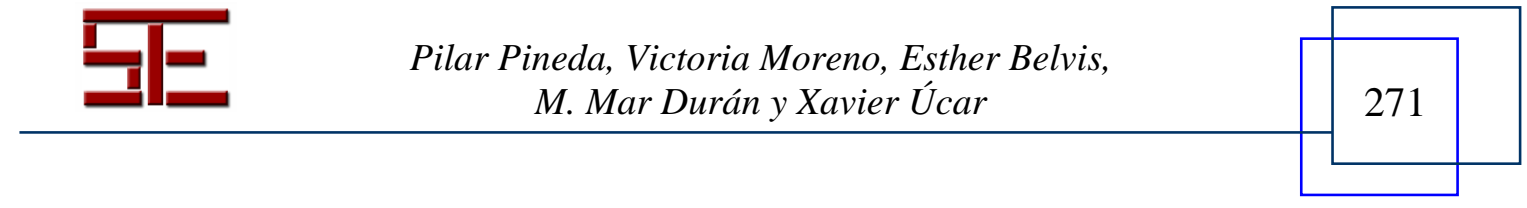




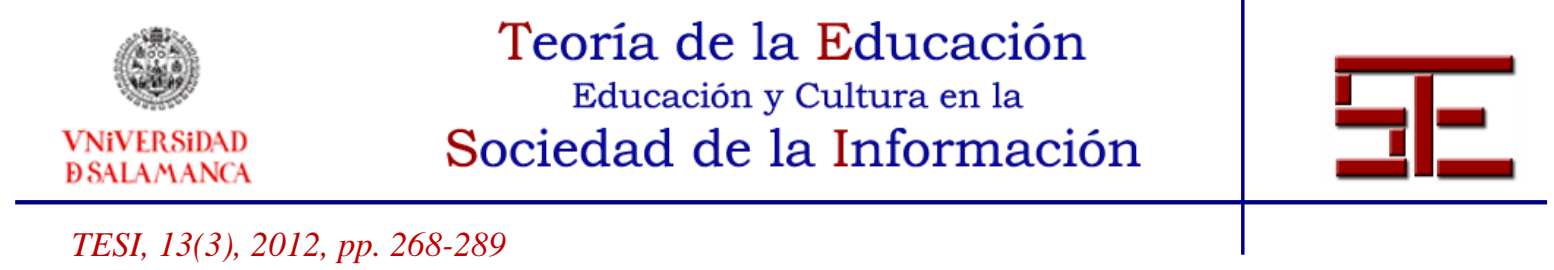

el apoyo social que se da en el puesto de trabajo hacia la aplicación de la formación. Ambos son aspectos relevantes para nuestro estudio.

En el sector sanitario se invierten muchos recursos para formar a los profesionales, con la intención de ayudarlos a realizar mejor sus funciones y a actualizar sus competencias para responder a las exigencias cambiantes de su profesión. La evaluación de dichas inversiones en formación es necesaria, ya que permite determinar cuáles son los resultados obtenidos, mostrando si la inversión ha sido eficaz o si es necesario introducir mejoras que aumenten la eficacia.

Los modelos de evaluación de la formación existentes plantean estrategias para evaluar la transferencia de acciones formativas concretas y proponen el diseño de instrumentos específicos para cada acción. Estos pretenden medir los cambios que las personas realizan en su desempeño profesional como resultado de la formación recibida. Una limitación de estos instrumentos es que su aplicación requiere mucho tiempo y recursos $\mathrm{y}$, al ser muy costosos, pocas organizaciones los aplican.

Frente a esta dificultad, las organizaciones optan por no evaluar la transferencia de sus planes de formación (Burke y Hutchins, 2008: 108), sino que, en el mejor de los casos, evalúan la transferencia de la acción formativa más estratégica, o encargan a agentes externos -consultores, investigadores, etc....- la realización de dicha evaluación. Es necesario dotar a los responsables de formación de instrumentos que les permitan afrontar esta función con eficacia.

Nuestro estudio es un ejemplo de escenario donde una organización busca la ayuda de expertos externos para evaluar la transferencia de uno de sus planes de formación, porque los resultados de esta formación son tremendamente estratégicos para ellos. En este caso, el Departamento de Salud del Gobierno catalán encarga a la entidad Unió Consorci Formació -UCF-, integrada por las dos principales asociaciones de centros sanitarios de Cataluña, el diseño y la implantación del Plan de Formación en Uso Racional del Medicamento, dirigido a todos los médicos de la región. El plan pretende mejorar las prácticas de prescripción farmacéutica de los médicos mediante la formación, y aumentar así la eficacia de los recursos públicos destinados a la sanidad. Nuestro equipo de investigación se encargó de evaluar la eficacia de dicho plan de formación en Uso Racional del Medicamento. Orientamos nuestra acción en la organización desde una perspectiva tanto teórica como práctica y desarrollamos una investigación dirigida a evaluar los resultados del plan de formación.

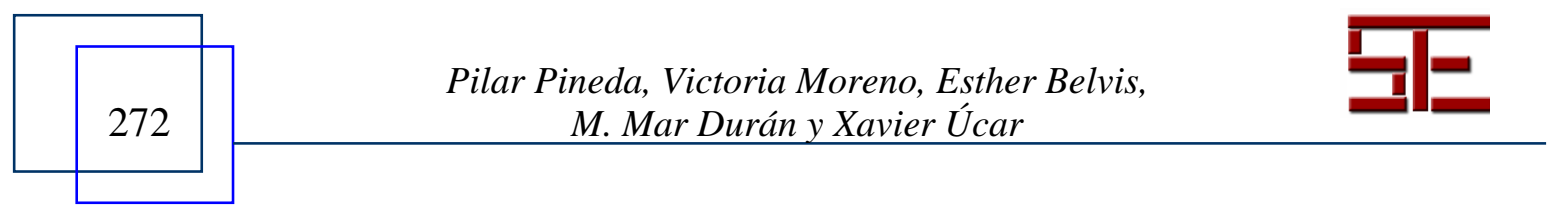




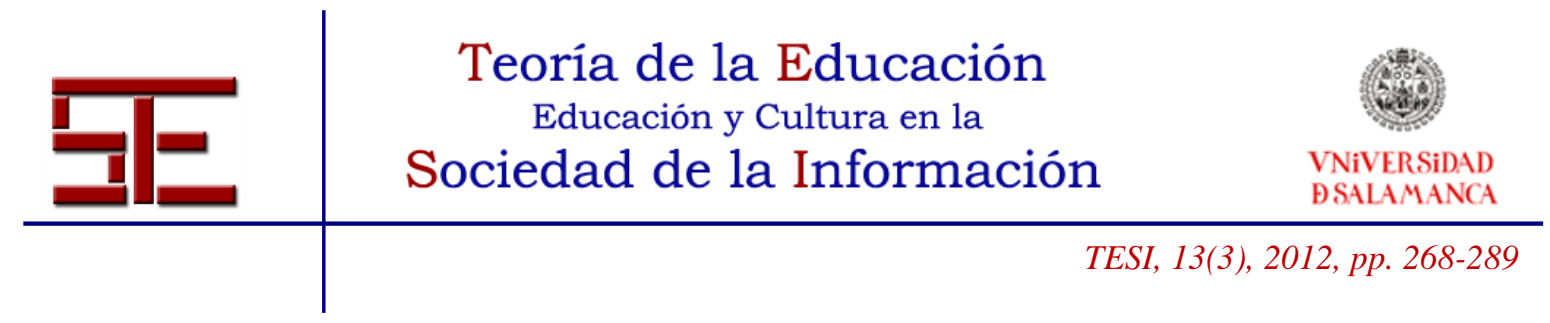

Dado que pretendíamos medir la transferencia de un plan de formación específico, nos propusimos las siguientes preguntas de investigación:

- ¿En qué medida el plan de formación logra los objetivos de aprendizaje y mejora profesional que se propone?

- ¿Qué cambios profesionales se dan como consecuencia de la participación en el plan de formación?

- ¿Qué resultados en el comportamiento profesional de los participantes en la formación se logran a partir de la formación en uso racional del medicamento?

- ¿Cuáles son las mayores barreras y los mayores catalizadores que afectan la transferencia de la formación al puesto de trabajo en el contexto del plan de formación en uso racional del medicamento?

\section{METODOLOGÍA}

\subsection{Planteamiento metodológico}

En esta investigación pretendemos evaluar los resultados del Plan de Formación en Uso racional del Medicamento. Por ello, nos planteamos los siguientes objetivos de evaluación:

1. Disseñar un plan de evaluación para la formación en el uso racional del medicamento.

2. Evaluar la satisfacción del participante con la formación realizada.

3. Evaluar los aprendizajes que los participantes adquieren con la formación.

4. Evaluar la transferencia de los aprendizajes al puesto de trabajo por parte de los participantes.

Para desempeñar la evaluación, se siguen tres fases:

- En primer lugar, analizamos el diseño, estructura y contenido del plan de formación. Se realizan diversas reuniones con las asociaciones encargadas de gestionar las acciones formativas que forman el Plan de Formación en Uso Racional del Medicamento, con la intención de recoger información sobre el plan de formación y las características de las distintas organizaciones implicadas en él.

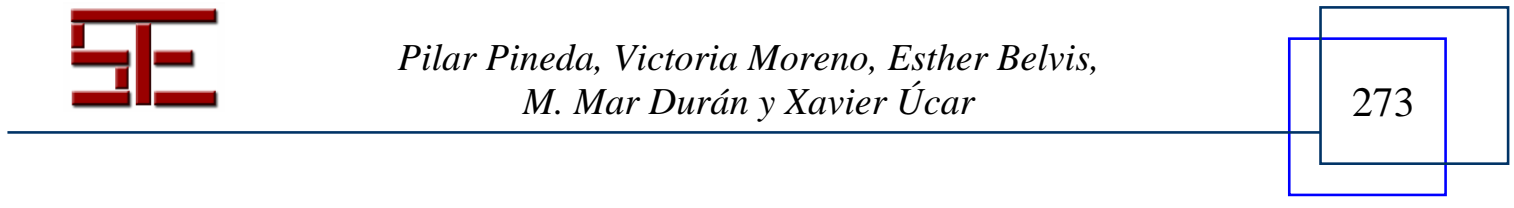




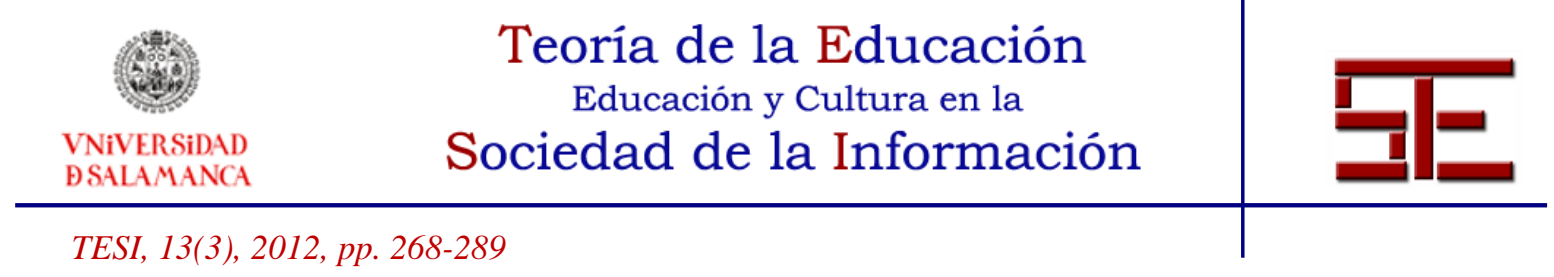

- En segundo lugar, creamos un sistema de evaluación como base para nuestra investigación. En este sentido, se tiene en cuenta que el plan de formación va dirigido a una población numerosa de más de mil trabajadores/as y que el sistema de evaluación debe ser implementado con pocos recursos.

- En tercer lugar, aplicamos el sistema de evaluación e interpretamos los resultados obtenidos.

\subsection{Escenario de la evaluación: el Plan de Formación en Uso Racional del Medicamento}

El plan de formación (UCF, 2007) que es objeto de evaluación se estructura en cuatro líneas formativas con los siguientes contenidos:

1. Maximización de beneficios y minimización de riesgos: integra los conceptos de mejora de la eficacia, la efectividad, la seguridad y la eficiencia.

- Actualización en farmacoterapéutica.

- Selección de medicamentos.

- Gestión integrada de la utilización de medicamentos.

- Gestión de los riesgos sanitarios derivados de la utilización de medicamentos.

- Educación sanitaria en materia de medicamentos.

2. Desarrollo de instrumentos de ayuda a la decisión clínica: elementos relevantes en la mejora de la calidad de la prescripción.

- Instrumentos de apoyo a la decisión clínica.

- Instrumentos de apoyo al análisis en la utilización de medicamentos.

- Formación en el manejo de nuevas tecnologías para la prescripción de medicamentos.

3. Comunicación y participación: integra el desarrollo de instrumentos y estrategias dirigidas a aumentar la participación de los profesionales en la formación.

- Desarrollo de un marco virtual de comunicación y participación de los profesionales en formación sobre uso de medicamentos.

- Desarrollo de actividades presenciales periódicas de comunicación y formación conjunta.

4. Evaluación y mejora de utilización de los medicamentos: integra las acciones dirigidas al desarrollo de proyectos de mejora en la utilización de los medicamentos.

- Evaluación de la utilización de medicamentos.

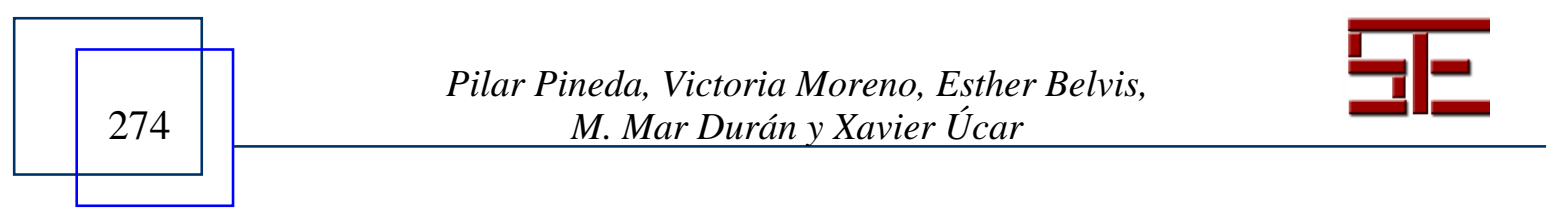




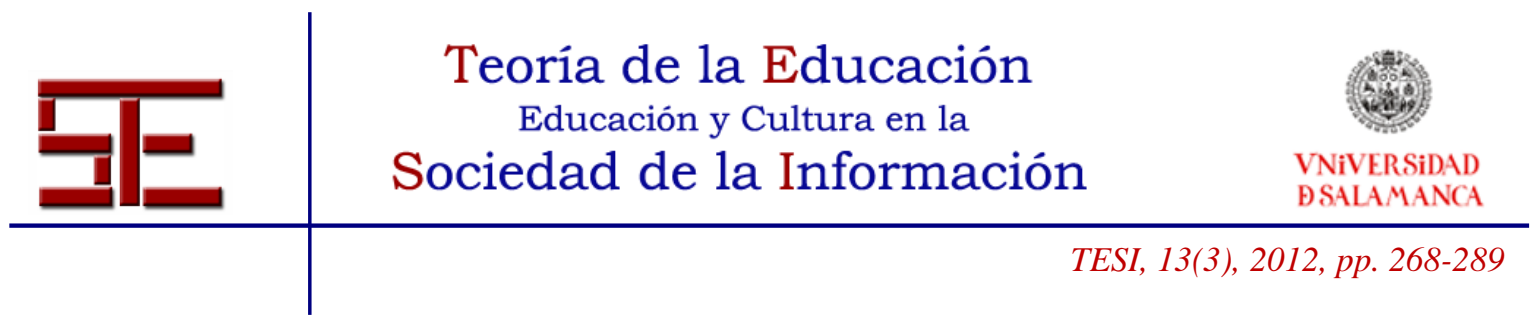

- Investigación en medicamentos: perspectiva clínica y asistencial.

- Mejora en la utilización de medicamentos.

Se realizaron un total de 153 acciones formativas entre abril y diciembre del 2007. Las acciones formativas fueron tanto cursos, como seminarios y talleres prácticos.

Como puede observarse se trata de un plan de formación de dimensiones considerables, al que se destina un presupuesto cuantioso. Su envergadura justifica que se evalúen sus resultados con rigor. Así se impulsa el proyecto 'Evaluación de la formación en el uso racional del medicamento', realizado por nuestro equipo de investigación. El proyecto se centra en evaluar la satisfacción y el aprendizaje de los facultativos médicos que participan en la formación, el nivel de transferencia al puesto de trabajo de los aprendizajes logrados y el impacto de la formación en los centros sanitarios. En este artículo presentamos la metodología utilizada para la evaluación, especialmente la referida a la transferencia de la formación y a los resultados de transferencia obtenidos, con el fin de responder a las preguntas de investigación formuladas.

\subsection{Sistema de evaluación: herramientas de evaluación y muestras}

La evaluación del Plan de Formación en materia del uso de Medicamentos parte del modelo holístico de evaluación (Pineda, 2002), y se estructura en dos acciones estratégicas con una metodología de trabajo diferente:

- Evaluación genérica. Se evalúa la satisfacción y la transferencia de una muestra representativa de acciones a través de dos cuestionarios genéricos.

- Evaluación específica. Se evalúan cinco acciones de formación a través de una metodología específica que incluye la evaluación de la satisfacción, del aprendizaje, de la adecuación pedagógica, de la transferencia y del impacto.

El siguiente esquema (Figura 1) ilustra los niveles e instrumentos correspondientes a cada estrategia evaluativa.

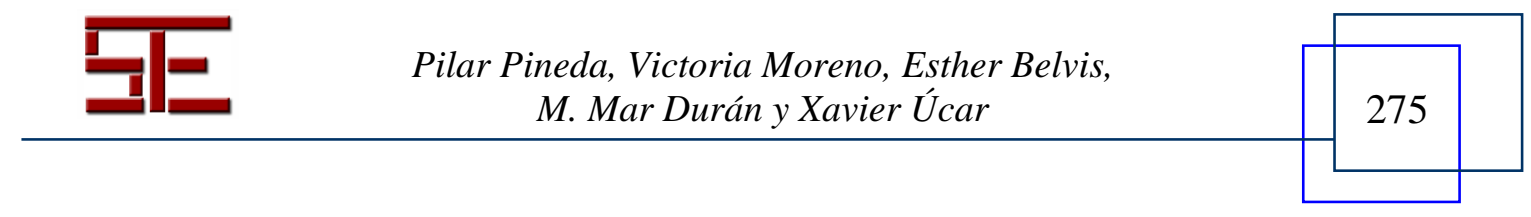


Figura 1. Estrategias de evaluación según niveles e instrumentos de evaluación

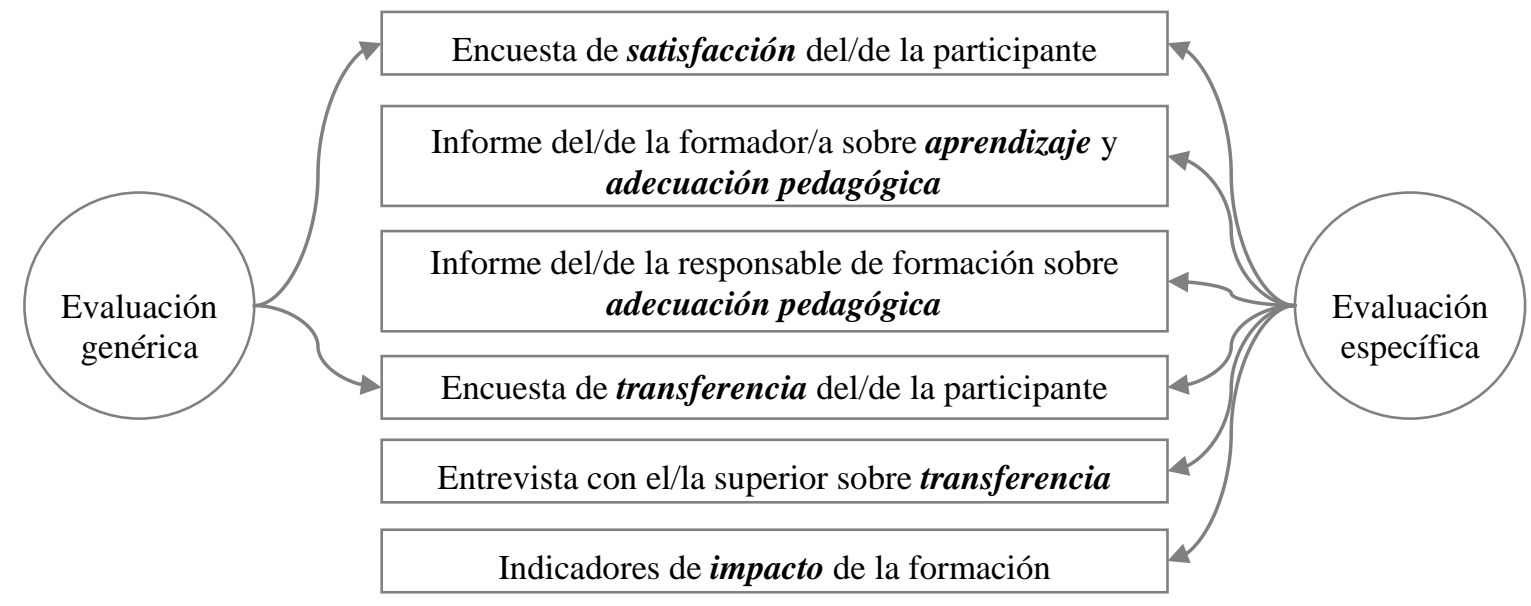

Como puede observarse en la Figura 1, la evaluación genérica, los resultados de la cual presentamos en este artículo, se centra en los niveles de satisfacción y transferencia. El Cuadro 1 ilustra los indicadores y variables que integran el plan de evaluación para estos dos niveles.

Cuadro 1. Indicadores y variables de las dimensiones satisfacción y transferencia

\begin{tabular}{|l|l|l|}
\hline $\begin{array}{l}\text { DIMENSION } \\
\text { ES }\end{array}$ & INDICADORES & VARIABLES \\
\hline \multirow{2}{*}{ PERFIL PARTICIPANTE } & $\begin{array}{l}\text {-Edad } \\
\text {-Sexo } \\
\text {-Tipo de acción formativa } \\
\text {-Sector/Centro de trabajo } \\
\text {-Ubicación territorial } \\
\text { - Nivel formativo }\end{array}$ \\
\cline { 2 - 3 } & OBJETIVOS & $\begin{array}{l}\text { - Logro } \\
\text { - Adaptación a las necesidades }\end{array}$ \\
\hline
\end{tabular}

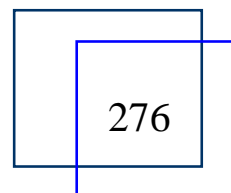

Pilar Pineda, Victoria Moreno, Esther Belvis, M. Mar Durán y Xavier Úcar 


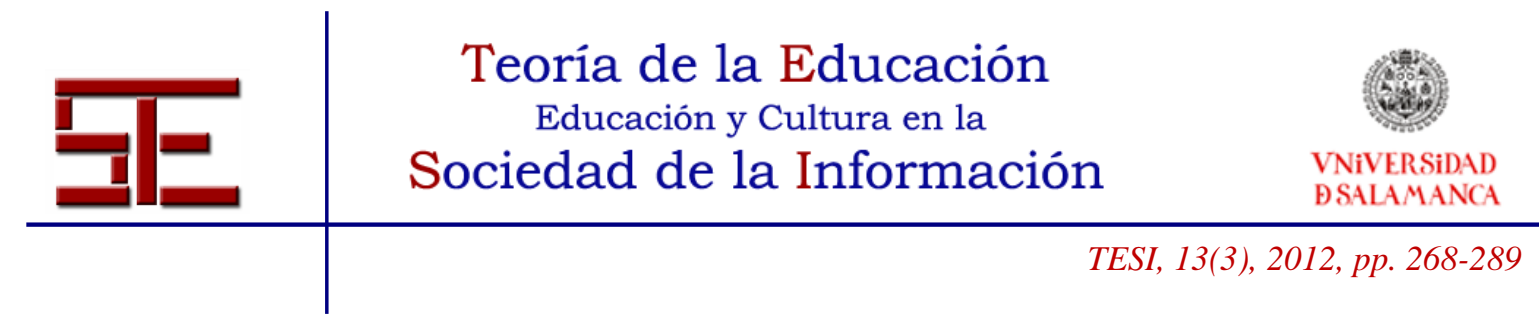

\begin{tabular}{|c|c|c|}
\hline \multirow{7}{*}{$\begin{array}{l}\text { SATISFAC- } \\
\text { CIÓN }\end{array}$} & CONTENIDOS & $\begin{array}{l}\text { - Interés } \\
\text { - Conocimientos previos } \\
\text { - Nivel de dificultad }\end{array}$ \\
\hline & METODOLOGÍA & $\begin{array}{l}\text { - Métodos utilizados } \\
\text { - Actividades desarrolladas } \\
\text { - Recursos didácticos }\end{array}$ \\
\hline & FORMADOR/A & $\begin{array}{l}\text { - Dominio contenidos } \\
\text { - Dominio metodológico } \\
\text { - Capacidad comunicativa } \\
\text { - Experiencia en el tema }\end{array}$ \\
\hline & ORGANIZACIÓN & $\begin{array}{l}\text { - Servicio administrativo } \\
\text { - Información recibida } \\
\text { - Horarios } \\
\text { - Calendario } \\
\text { - Instalaciones }\end{array}$ \\
\hline & CLIMA & $\begin{array}{l}\text { - Participación del grupo } \\
\text { - Cohesión del grupo } \\
\text { - Relación formador- } \\
\text { participantes }\end{array}$ \\
\hline & EVALUACIÓN & $\begin{array}{l}\text { - Presencia } \\
\text { - Tipo } \\
\end{array}$ \\
\hline & $\begin{array}{l}\text { APLICACIÓN Y } \\
\text { VALORACIÓN GLOBAL }\end{array}$ & $\begin{array}{l}\text { - Aplicación aprendizajes } \\
\text { - Logro de expectativas } \\
\text { - Utilidad en la práctica }\end{array}$ \\
\hline
\end{tabular}

\begin{tabular}{|l|l|l|}
\hline & - Tipología (conocimientos, \\
APRENDIZAJES & actitudes, procedimientos) \\
APLICADOS & $\begin{array}{l}\text { - Volumen } \\
\text { - Autopercepción del cambio }\end{array}$ \\
\hline
\end{tabular}

Pilar Pineda, Victoria Moreno, Esther Belvis, M. Mar Durán y Xavier Úcar 


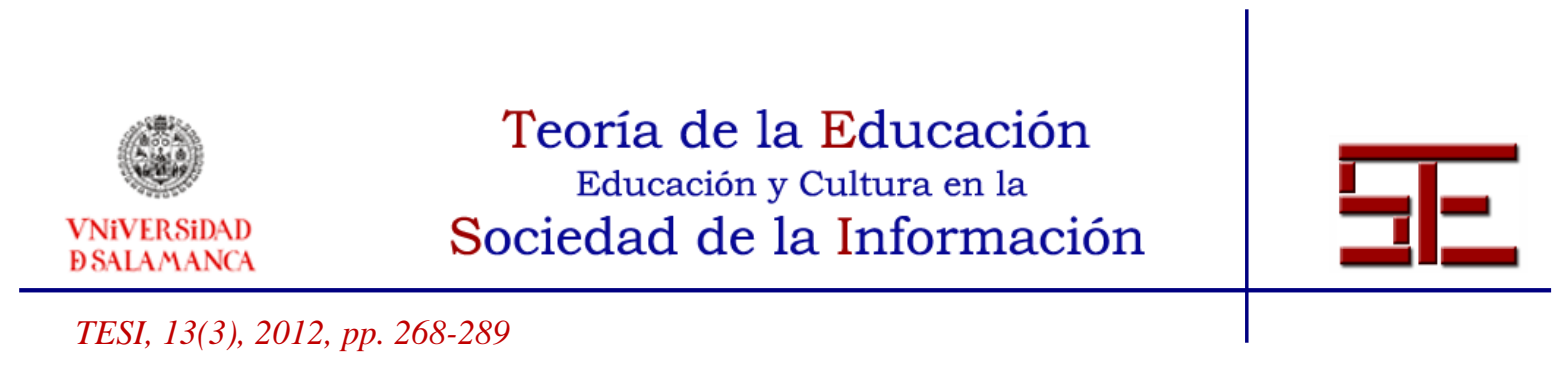

\begin{tabular}{|c|c|c|}
\hline \multirow[t]{3}{*}{$\begin{array}{l}\text { TRANSFE- } \\
\text { RÈNCIA }\end{array}$} & $\begin{array}{l}\text { CONDICIONANTES } \\
\text { ORGANIZATIVOS }\end{array}$ & $\begin{array}{l}\text { - Cultura organizativa } \\
\text { (abierta/cerrada al cambio) } \\
\text {-Apoyo y seguimiento (del } \\
\text { formador, jefe, compañeros) } \\
\text { - Disponibilidad de recursos } \\
\text { (materiales, instalaciones...) } \\
\text { - Clima en la organización (interés, } \\
\text { participación...) }\end{array}$ \\
\hline & $\begin{array}{l}\text { CONDICIONANTES } \\
\text { PEDAGÓGICOS }\end{array}$ & $\begin{array}{l}\text { - Diseño de la formación } \\
\text { (aplicabilidad) } \\
\text { - Orientación a la transferencia } \\
\text { (estrategias utilizadas) }\end{array}$ \\
\hline & $\begin{array}{l}\text { CONDICIONANTES } \\
\text { PERSONALES }\end{array}$ & $\begin{array}{l}\text { - Preparación y competencias de los } \\
\text { participantes } \\
\text { - Motivación para transferir }\end{array}$ \\
\hline
\end{tabular}

En relación a las fuentes de información, la evaluación de la transferencia en el enfoque genérico utiliza como fuente principal de información a los participantes en la formación. Esta opción responde a varios motivos. En primer lugar, los profesionales del sector sanitario se caracterizan por una alta autonomía en el ejercicio de sus funciones, por lo que se convierten en la fuente de información más importante sobre los cambios que la formación genera en su trabajo. En segundo lugar, la evaluación cuantitativa de un programa tan amplio y variado como el que nos ocupa exige necesariamente la utilización del participante como fuente de información, dada la dificultad y el coste del acceso a otros colectivos como el superior o los compañeros. En tercer lugar, la evaluación específica realizada, con un enfoque más cualitativo, permite contrastar los resultados cuantitativos obtenidos y el hecho de que ambos coincidan muestra la rigurosidad y validez de estos últimos. Por último, con la evaluación específica realizada, a través de entrevistas a los superiores, se han recogido evidencias sólidas de la transferencia e impacto de la formación; estas evidencias permiten matizar los resultados cuantitativos obtenidos. La información sobre esta parte más cualitativa del estudio será objeto de otras publicaciones

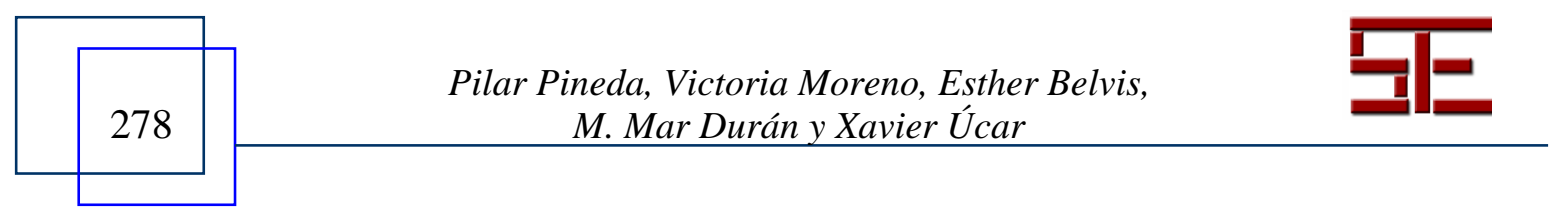




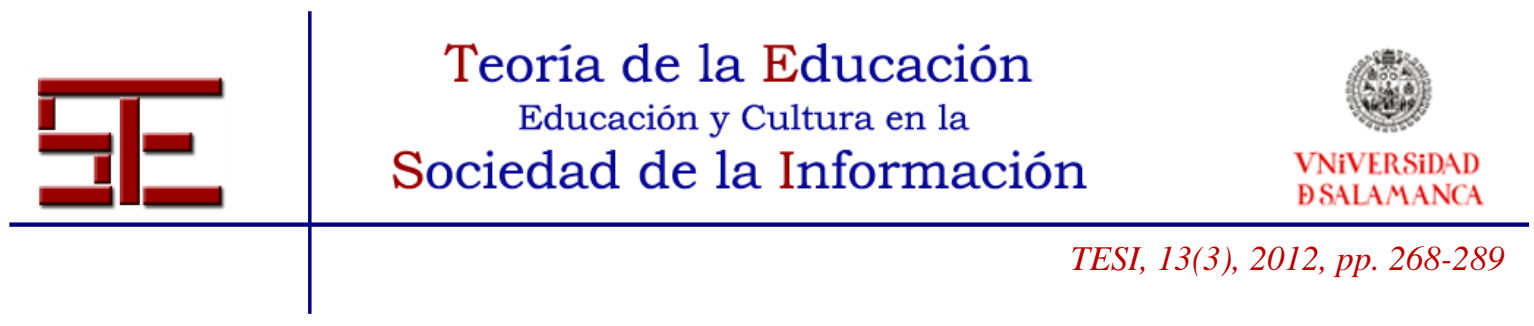

En relación a los instrumentos de evaluación, la evaluación de la transferencia en la estrategia genérica se realiza mediante un cuestionario dirigido al participante. Se considera que este instrumento es el más efectivo, dado el tamaño de la población, su dispersión en el territorio y su facilidad de aplicación por parte del departamento de formación en un futuro.

Para dar respuesta a este objetivo de evaluación se diseña un cuestionario ad hoc que aborda los siguientes ámbitos, ya mencionados en el plan de evaluación:

Perfil profesional: recoge información referida a las características personales (edad, género...) así como de aspectos referidos a su puesto de trabajo (lugar, tipología de centro...). Estas variables independientes permiten evaluar la transferencia a través de colectivos o grupos específicos.

Aprendizajes alcanzados: recoge información sobre la tipología de aprendizajes que se han realizado y el grado en que se han alcanzado, así como el grado de respuesta de la formación a las necesidades de los participantes. Para diseñar las preguntas de este apartado se revisó el plan de formación teniendo en cuenta los principales objetivos formativos que se pretendían conseguir.

Aplicación de la formación: recoge información referida a la tipología y grado de mejoras producidas en la actuación profesional. Las mejoras se relacionan directamente con los aprendizajes realizados en la formación, y se refieren a las competencias que se han aplicado en el puesto de trabajo: la selección, homogenización de criterios y uso de medicamentos, la gestión de la información, la relación médico-paciente, la introducción de protocolos, la incorporación de nuevos instrumentos, etc.

Asimismo se valoran aspectos vinculados al apoyo a la aplicación de la formación a través de diferentes agentes, en concreto del formador y del superior directo.

Factores que afectan la aplicación: recoge información tanto sobre aspectos vinculados a aspectos personales (motivación, expectativas...) como de la organización (clima, recursos disponibles...) que tienen incidencia en la transferencia de los aprendizajes realizados. Del conjunto de factores que aparecen en la literatura (Noe, 1996; Holton 2005. Burke-Hutchins; 2008) se han seleccionado estos por ser los más relevantes en otros estudios y los que se adaptan mejor a las características del sector sanitario.

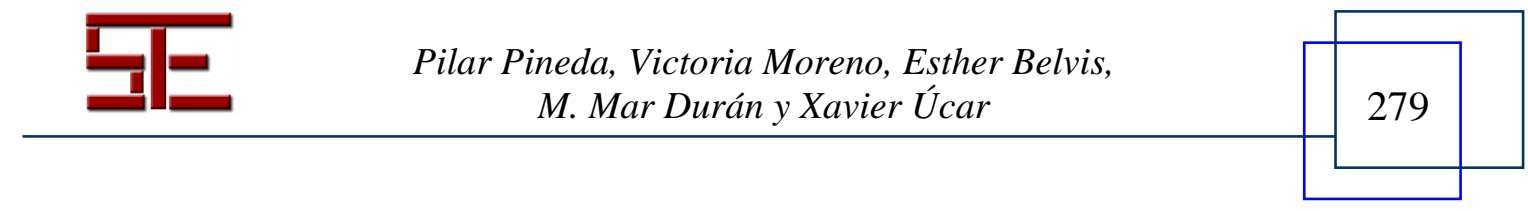




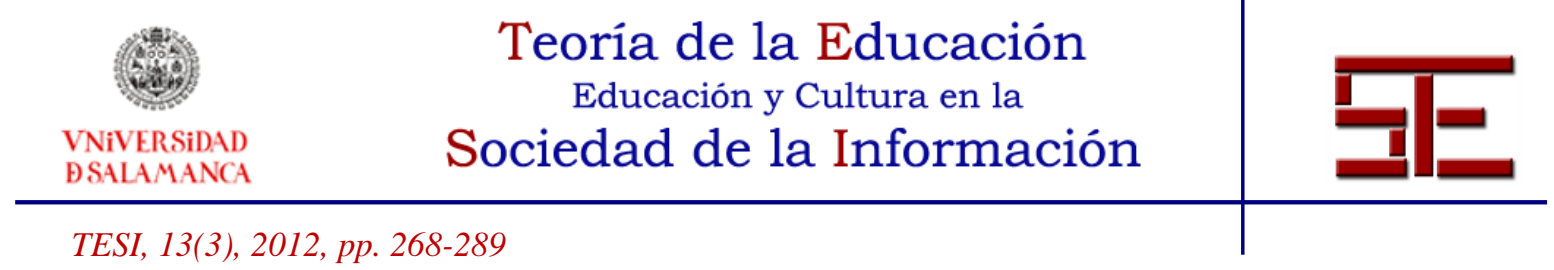

El cuestionario de transferencia fue sometido a una validación de contenido mediante el sistema de jueces. En el proceso participan tanto validadores expertos en metodología como expertos en formación del sector sanitario. Las aportaciones de los expertos del sector permitieron identificar las competencias desarrolladas con la formación, ajustando así el instrumento para la evaluación de la transferencia. Los resultados obtenidos con puntaciones altas en la mayoría de ítems, y con una clara univocidad y pertinencia en la mayoría de casos, indican una clara adecuación del contenido y, por tanto, poca necesidad de introducir cambios, lo que aporta garantías de validez y fiabilidad.

La aplicación del cuestionario se realiza a través de un aplicativo on-line diseñado por un técnico experto. Para recoger el máximo de información se realiza un muestreo por oportunidad, es decir, se ofrece la posibilidad de contestar el cuestionario a todos los participantes. Finalmente la muestra se configura a partir de los cuestionarios recibidos. En total se obtuvo una muestra de 351 cuestionarios de 53 acciones formativas diferentes, de las 153 que se realizaron (Cuadro 2).

Cuadro 2. Acciones formativas y participantes

\begin{tabular}{|l|c|c|}
\hline Línea formativa & $\begin{array}{l}\text { Frecuencia } \\
\text { de acciones }\end{array}$ & Sujetos \\
\hline $\begin{array}{l}\text { Maximización de beneficios y minimización de } \\
\text { riesgos }\end{array}$ & 5 & 30 \\
\hline Desarrollo y mejora de instrumentos & 11 & 61 \\
\hline $\begin{array}{l}\text { Evolución y mejora de la utilización de los } \\
\text { medicamentos }\end{array}$ & 35 & 228 \\
\hline Comunicación y participación & 2 & 3 \\
\hline
\end{tabular}

Para recoger la información se cuelga el aplicativo on-line en el servidor y se hace llegar el link a los diferentes participantes en las acciones formativas de la muestra, a través del correo electrónico. Se ha hecho un seguimiento exhaustivo del índice de respuesta de todas las acciones formativas enviando recordatorios a los participantes.

Los datos del cuestionario de transferencia se vacían posteriormente en una matriz Excel con las diferentes variables definidas y codificadas previamente. Para la explotación de los datos del cuestionario se realizan diversos análisis de la varianza de

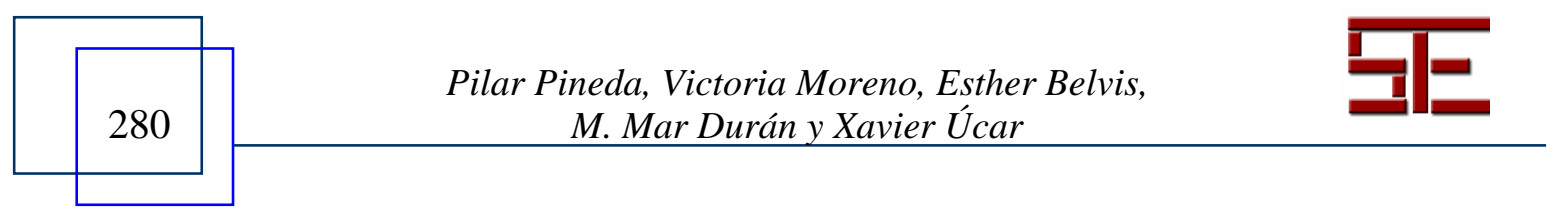




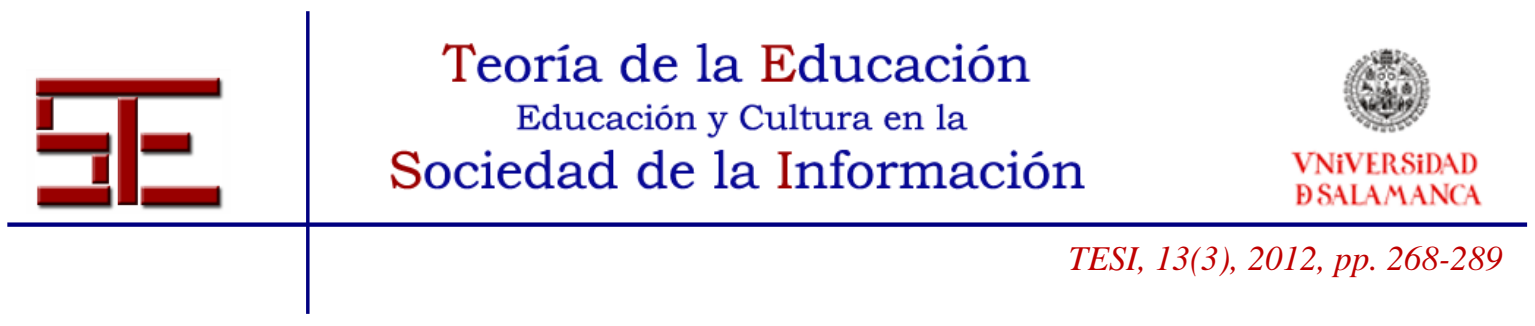

las principales variables dependientes tomando como variable independiente el "centro de trabajo" del/de la participante.

Los resultados obtenidos se plasman en un informe de evaluación, estructurado siguiendo las dimensiones de evaluación, que se entrega al cliente: el equipo directivo de UCF, como máximo responsable del plan de formación evaluado.

\section{RESULTADOS}

Las personas que han contestado el cuestionario son en un $60,6 \%$ mujeres y en un $39,4 \%$ hombres, mayoritariamente con edades comprendidas entre los 30 y los 50 años (70\%), con un nivel de estudios de licenciatura. En relación a los datos descriptivos de la variable independiente, la mayoría de personas trabajan en Centros de Atención Primaria (44,6\%). A continuación se encuentran los profesionales que desarrollan su tarea en hospitales $(21,2 \%)$, en centros socio-sanitarios $(19,4 \%)$ y de salud mental $(11,7)$. Los centros del ámbito de la dependencia $(3,1 \%)$ son los que han participado en menor grado en la formación.

Los participantes en las acciones formativas consideran que la formación responde adecuadamente a las necesidades formativas en un grado del 3,82 y que el nivel de aprendizaje alcanzado se sitúa en un 3,36 en una escala de 0 a 5. Los resultados confirman que hay diferencias significativas en la respuesta a las necesidades formativas según el centro de trabajo, $F(4,317)=2,73, p=.03$. De acuerdo con la prueba de seguimiento de Tukey -aplicada al nivel de .05-, concretamente se dan diferencias significativas entre los centros de Atención Especializada $(4,04)$ y los centros de Atención primaria $(3,65)$.

Se pregunta a las personas participantes en la formación cuáles de los objetivos de aprendizaje del plan de formación han alcanzado en las acciones formativas cursadas. La mayoría de participantes manifiestan haber alcanzado aprendizajes en el uso de medicamentos $(91,1 \%)$ y en la actualización de conocimientos médicos $(90,6 \%)$, aprendizajes que se relacionan con la línea de formación que tiene más presencia en la muestra. Otros aprendizajes que también se deben tener en cuenta son el establecimiento de protocolos de actuación $(79,8 \%)$, la homogenización de criterios $(78,2 \%)$ y aquellos que giran en torno a la evaluación $(77,6 \%)$. Contrariamente los aspectos en los que ha habido menos aprendizaje están vinculados con la investigación experimental $(37,1 \%)$, las habilidades comunicativas $(45,9 \%)$ y las tecnologías de la

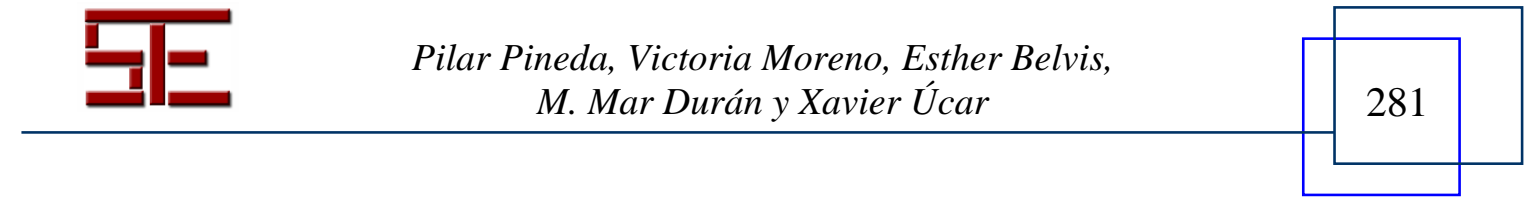




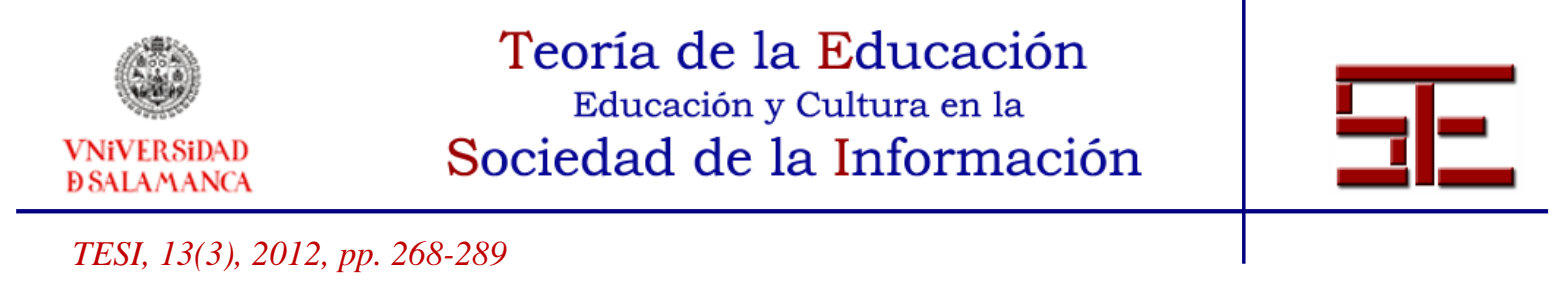

información y la comunicación $(57,8 \%)$. No obstante, el grado de aprendizaje medio logrado es del 3,36 en una escala de 0 a 5, y vale la pena mencionar que no se dan diferencias en las puntuaciones entre los distintitos tipos de centro de trabajo $F(4,294)$ $=2,45, p=.05$.

En referencia a la aplicación de la formación, los participantes consideran, en una escala de 0 a 5 , que la formación ha incidido de forma positiva en el desarrollo personal $(3,28) \mathrm{y}$, en menor medida, que ha posibilitado la introducción de cambios en la actuación de los profesionales ha permitido la actuación profesional $(2,99)$. Este resultado sorprende ya que la formación pretende incidir principalmente en la actuación profesional. Al mismo tiempo, las dos variables muestran una alta desviación típica $(1,031$ y 1,182) cosa que indica cierta disparidad en las valoraciones de los participantes. Los resultados obtenidos muestran que no hay diferencias significativas entre los centros de trabajo ni en la incidencia de la formación en el desarrollo personal, $F(4,291)=1,05, p=.38$, ni en modificación de la actuación de los profesionales, $F(4$, 292) $=1,07, p=.42$.

Lo que se detecta en ambos casos es una elevada desviación típica, sobre todo, en los centros de Atención primaria $(1,204)$ y socio-sanitarios $(1,261)$ en la modificación de la actuación profesional. Este resultado también se ha observado en la evaluación específica donde se observa que un tiempo después de finalizar la formación los participantes dan opiniones más realistas y matizadas sobre las posibilidades de transferencia. En este sentido apuntan a que las condiciones de trabajo con una alta carga asistencial y falta de tiempo dificultan en algunos puestos de trabajo y organizaciones la transferencia.

Cuando se pregunta a los participantes sobre las mejoras en la actuación profesional gracias a la formación un $82,1 \%$ de los participantes considera que las mejoras en la actuación profesional han estado relacionadas principalmente con el establecimiento de procesos de selección y utilización de medicamentos. En menor medida, se han producido mejoras en la homogenización de criterios $(64,8)$, en el funcionamiento general de la organización (59\%) y en la gestión de la información $(58,3 \%)$. Por otro lado, los procesos en los que se han detectado menos mejoras son: los sistemas de detección e intervención (46\%), en la relación médico-paciente $(44,7 \%)$ y en el acceso a la información $(43,9 \%)$.

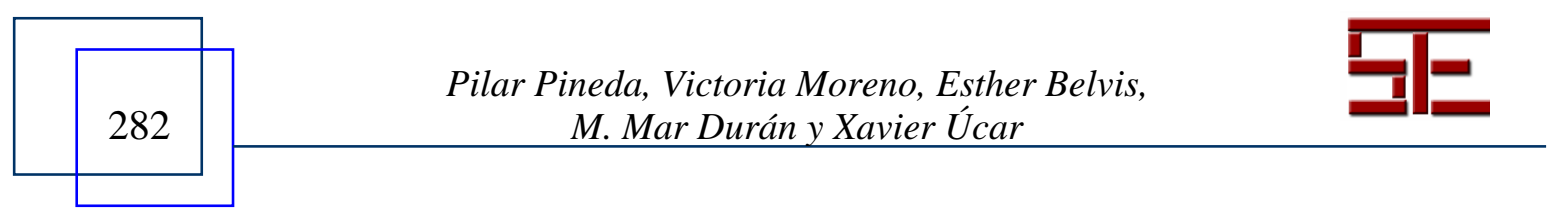




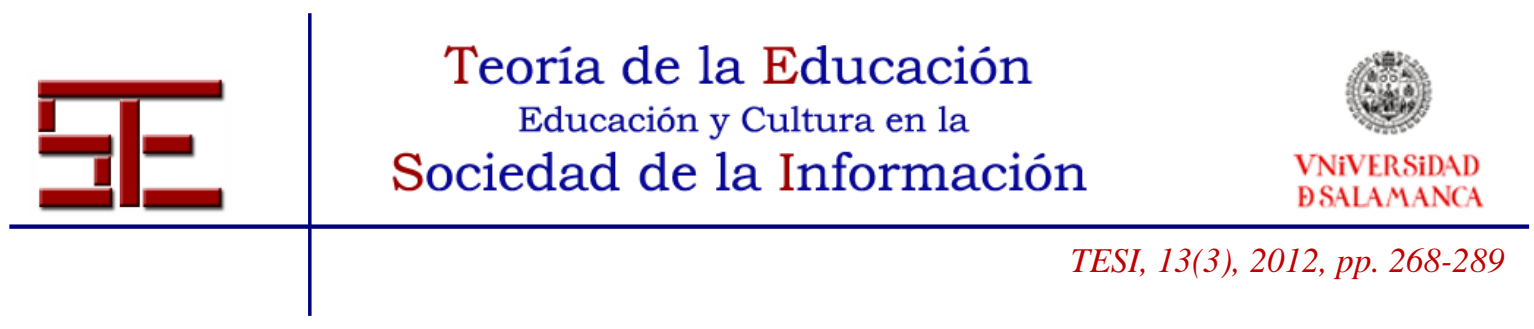

En términos más amplios, se pide también que los participantes valoren el nivel de aplicación de la formación. Los resultados muestran como el nivel de aplicación de la formación tiene una puntuación de un 3,1 (desviación típica 1,033). Este resultado es un tanto más positivo que el resultado sobre el efecto de la formación en la actuación profesional, aunque ambas cuestiones están relacionadas. De nuevo, los resultados muestran que no hay diferencias significativas en la variable nivel de aplicación de la formación entre los diferentes centros de trabajo, $F(4,277)=, 43, p=.78$.

En lo que se refiere al apoyo recibido por parte del formador y del responsable de la formación en la aplicación de la formación al puesto de trabajo, la puntuación media obtenida por el formador es de 3,52 (desviación típica 1,030), ligeramente superior a la media obtenida por el superior directo, 3,17 (desviación típica 1,182). La alta desviación típica en los ítems hace suponer que diferentes factores vinculados con el participante, el puesto de trabajo o la formación generan estas diferencias.

En referencia al apoyo recibido, se dan diferencias entre los centros al respecto de la persona formadora, $F(4,277)=2,55, p=.04$, no así al respecto del superior directo, $F(4,277)=1,34, p=.26$. Las diferencias se dan entre los centros socio-sanitarios $(3,85)$, donde los participantes manifiestan un mayor apoyo del formador, y los centros de atención primaria $(3,37)$, donde el apoyo percibido es significativamente mayor. Estas diferencias entre los centros pueden estar condicionadas tanto por la categoría laboral de los participantes como por la organización interna de los centros y su política de formación. En este caso, dada la heterogeneidad de las acciones formativas y de sus participantes resulta complicado establecer la principal causa pero, sí se pueden apuntar hipótesis sobre aspectos que pueden haber influido en el nivel de transferencia.

Un aspecto importante de la evaluación es la identificación de los factores que determinan la transferencia, para explicar los motivos del nivel de eficacia alcanzado con la formación e identificar estrategias que ayuden a mejorarla. En este sentido, destacan las aportaciones de Holton (2005) porque muestran los factores de la transferencia más relevantes.

Se pregunta a los participantes en la formación sobre los siguientes factores que afectan a la transferencia de la formación: autoeficacia, motivación, apoyo de los compañeros/as, apoyo de la formación en la organización, clima de trabajo positivo, autonomía, simplicidad en la aplicación de la formación, disponibilidad de tiempo para

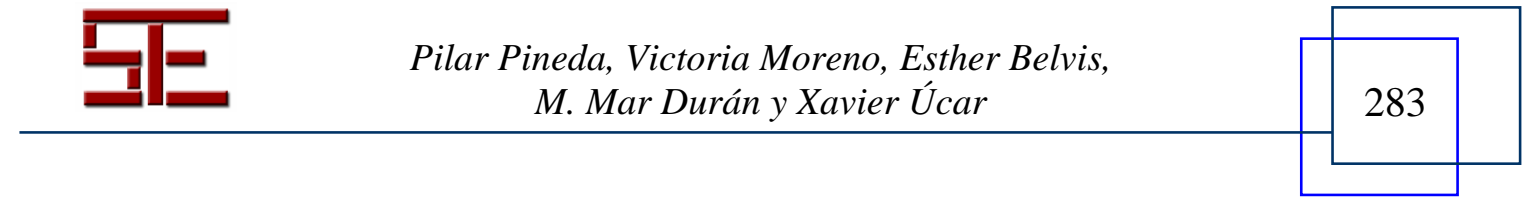




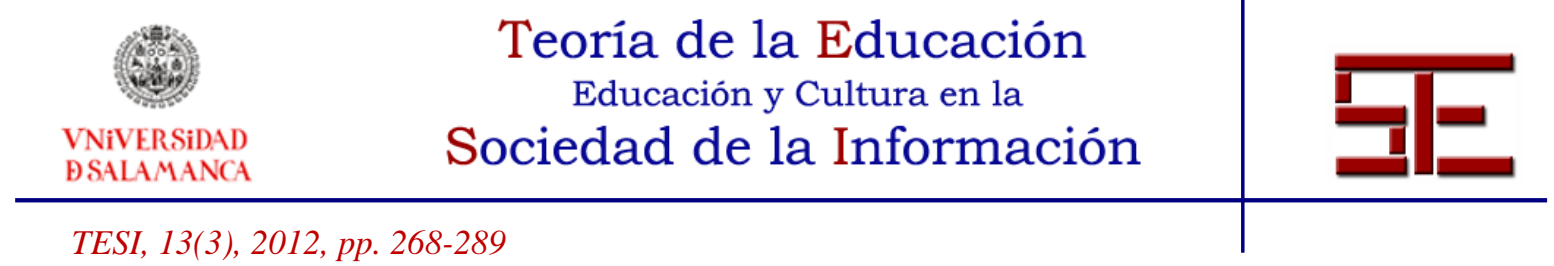

la aplicación, existencia de los recursos necesarios para la aplicación y oportunidades de implementación.

Las personas encuestadas consideran que los factores que más facilitan su transferencia de la formación al puesto de trabajo son primeramente el apoyo de los compañeros/as (3,68 en una escala de 0 a 5), seguido de la motivación $(3,64)$ y el clima positivo en el puesto de trabajo $(3,55)$. Otros factores aparecen también como catalizadores de la transferencia de la formación, pero de un modo más moderado, los cuales son el apoyo de la organización $(3,49)$, la autonomía $(3,33)$, la autoeficacia $(3,32)$ y oportunidades de aplicación $(3,16)$. Los aspectos que son barreras para la aplicación de la formación son: simplicidad en la aplicación de la formación $(2,55)$ y la existencia de los recursos necesarios para transferir la formación. Este resultado es interesante y sigue la tendencia de otros estudios, ya que muy a menudo estos factores apuntados por los/las participantes son obstáculos para la transferencia (Jhpiego, 2002; Lim \& Johnson, 2002; Pineda y otros, 2007; Pineda y otros, 2008). El Cuadro 3 muestra las medias y desviaciones típicas para todos los factores según centro de trabajo.

Cuadro 3. Media y desviación típica de los factores que afectan la transferencia de la formación por tipo de centro

\begin{tabular}{|c|c|c|c|c|c|c|c|c|c|c|c|c|}
\hline \multirow{3}{*}{$\begin{array}{l}\text { Factores que } \\
\text { afectan la } \\
\text { transferencia } \\
\text { de la formación }\end{array}$} & \multicolumn{12}{|c|}{ Centro de trabajo } \\
\hline & \multicolumn{2}{|c|}{$\begin{array}{l}\text { Atención } \\
\text { primaria }\end{array}$} & \multicolumn{2}{|c|}{$\begin{array}{l}\text { Atención } \\
\text { especiali- } \\
\text { zada } \\
\end{array}$} & \multicolumn{2}{|c|}{$\begin{array}{l}\text { Salud } \\
\text { mental }\end{array}$} & \multicolumn{2}{|c|}{$\begin{array}{l}\text { Socio- } \\
\text { sanitario }\end{array}$} & \multicolumn{2}{|c|}{$\begin{array}{l}\text { Dependen- } \\
\quad \text { cia }\end{array}$} & \multicolumn{2}{|c|}{ Total } \\
\hline & $M$ & $D T$ & $M$ & $D T$ & $M$ & $D T$ & $M$ & $D T$ & $M$ & $D T$ & $M$ & $D T$ \\
\hline $\begin{array}{l}\text { Oportunidades } \\
\text { de aplicación }\end{array}$ & 2.97 & 1,19 & 3.45 & 1,15 & 3.48 & ,78 & 3.12 & 1,02 & 3.00 & 1,50 & 3.16 & 1,13 \\
\hline $\begin{array}{l}\text { Recursos } \\
\text { necesarios }\end{array}$ & 2.79 & 1,14 & 3.21 & 1,12 & 3.00 & 1,13 & 3.14 & ,99 & 2.78 & 1,48 & 2.97 & 1,12 \\
\hline $\begin{array}{l}\text { Tiempo } \\
\text { disponible para } \\
\text { la aplicación }\end{array}$ & 2.34 & 1,25 & 2.43 & 1,32 & 3.10 & 1,08 & 2.88 & 1,14 & 2.22 & 1,20 & 2.55 & 1,25 \\
\hline $\begin{array}{l}\text { Simplicidad en } \\
\text { la aplicación de } \\
\text { la formación }\end{array}$ & 2.44 & 1,24 & 2.51 & 1,27 & 2.41 & 1,21 & 2.50 & 1,23 & 2.00 & 1,12 & 2.45 & 1,23 \\
\hline
\end{tabular}

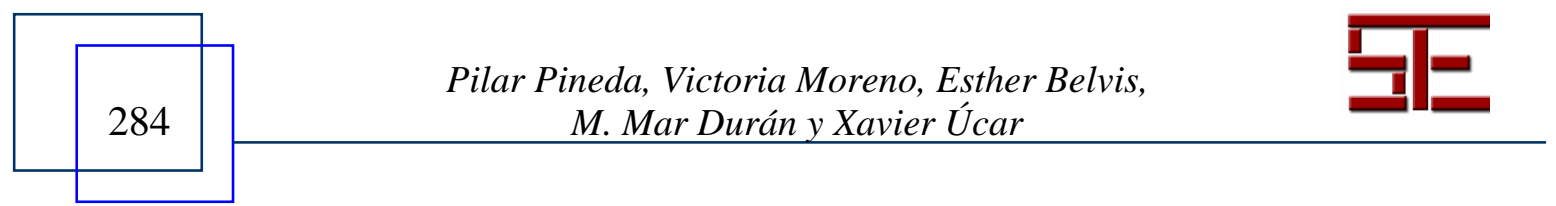




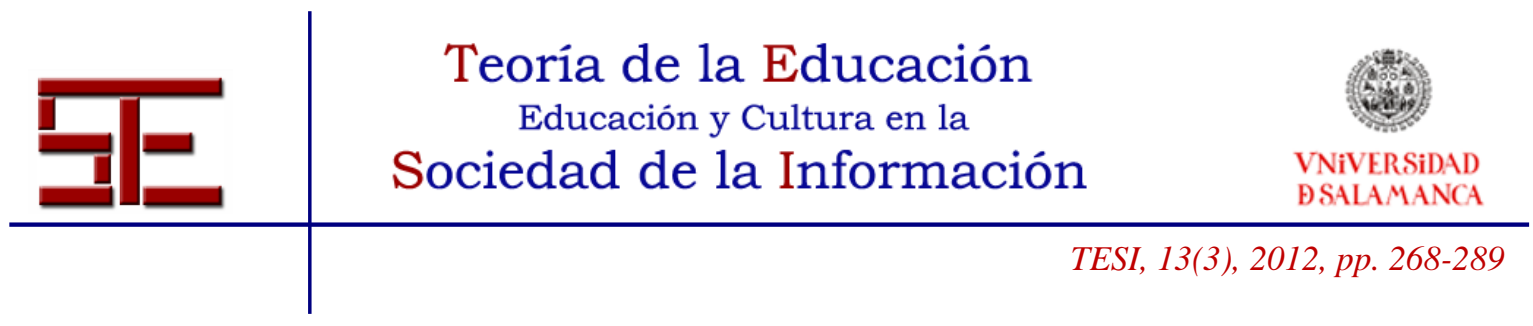

\begin{tabular}{|l|r|r|r|r|r|r|r|r|r|r|r|r|}
\hline Autonomía & 3.28 & 1,24 & 3.66 & 1,06 & 3.48 & 1,09 & 3.34 & 1,06 & 2.89 &, 93 & 3.38 & 1,15 \\
\hline $\begin{array}{l}\text { Clima de } \\
\text { trabajo positivo }\end{array}$ & 3.52 & 1,18 & 3.38 & 1,13 & 3.93 &, 88 & 3.62 & 1,01 & 3.33 & 1,50 & 3.55 & 1,12 \\
\hline $\begin{array}{l}\text { Apoyo de la } \\
\text { organización }\end{array}$ & 3.45 & 1,17 & 3.28 & 1,25 & 3.66 &, 97 & 3.74 &, 92 & 3.33 & 1,12 & 3.49 & 1,12 \\
\hline $\begin{array}{l}\text { Apoyo de los } \\
\text { compañeros/as }\end{array}$ & 3.66 & 1,14 & 3.62 & 1,18 & 3.86 & 1,16 & 3.70 & 1,02 & 3.44 & 1,33 & 3.68 & 1,13 \\
\hline Motivación & 3.60 & 1,09 & 3.70 &, 97 & 3.86 & 1,03 & 3.58 & 1,13 & 3.56 & 1,13 & 3.64 & 1,06 \\
\hline Autoeficacia & 3.19 & 1,12 & 3.51 & 1,03 & 3.28 &, 96 & 3.48 &, 81 & 3.11 & 1,62 & 3.32 & 1,05 \\
\hline
\end{tabular}

M = Media; DT = Desviación Típica

Los resultados obtenidos muestran que es posible evaluar la transferencia de la formación en una población grande de participantes mediante instrumentos cuantitativos, aunque ello tiene una serie de implicaciones y condicionantes. La principal condición es que la formación desarrolle un bloque de competencias homogéneo en todos los participantes, de manera que se puedan obtener resultados sobre los cambios en dichas competencias mediante un cuestionario. Otra implicación es la dificultad para obtener información del superior o de los compañeros, dado el coste y las dificultades logísticas que ello supone; esta implicación puede superarse introduciendo instrumentos cualitativos, como se ha realizado en la evaluación específica de cinco acciones formativas. Este enfoque cuantitativo tiene también implicaciones positivas, ya que permite el fácil acceso a la información con poco coste y la generalización de los resultados. Estos aspectos se desarrollan con más detalle en la discusión.

\section{DISCUSIÓN Y CONCLUSIONES}

Los resultados obtenidos muestran que los profesionales del sector sanitario participantes en el Plan de formación en Uso Racional del Medicamento realizan una valoración positiva de las acciones formativas realizadas. Consideran que es una formación útil, que responde a sus expectativas y que los aprendizajes realizados son aplicables a la práctica profesional. La mayoría de participantes ha introducido mejoras en su actuación profesional, especialmente en las funciones relacionadas con la selección y utilización de medicamentos. Este era uno de los principales objetivos de la formación, por lo que se puede afirmar que la formación ha sido eficaz al modificar la

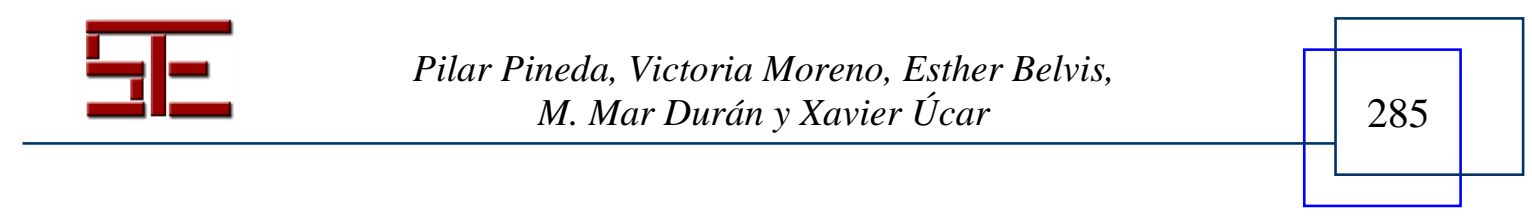




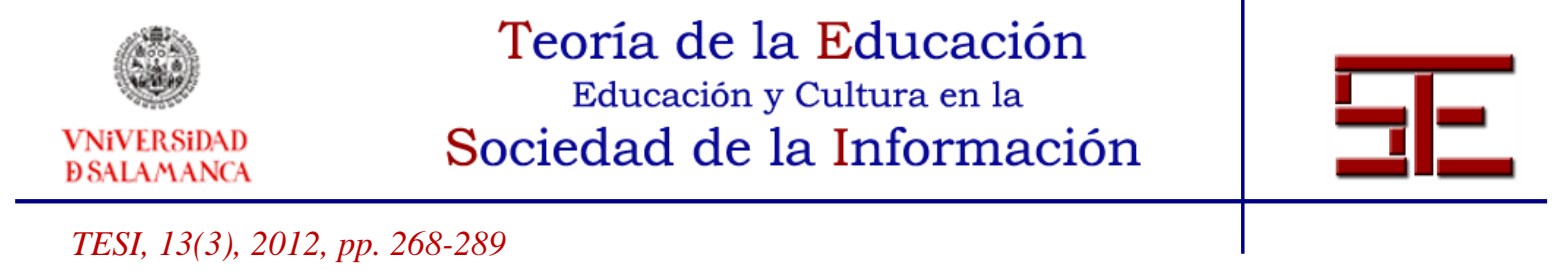

actuación profesional de los médicos en el uso de medicamentos. Por otro lado, los resultados muestran que en otras competencias, como el uso de sistemas de detección e intervención, o en la relación médico-paciente, las mejoras en la actuación profesional se dan en menos de la mitad de los médicos evaluados. Este resultado, similar a los resultados de otras investigaciones (Saks \& Belcourt, 2006), muestra un área de mejora importante, bien sea en el diseño y desarrollo de la formación, o bien en otros factores del contexto que pueden obstaculizar la transferencia.

El sistema de evaluación diseñado también recoge información sobre los factores que condicionan la transferencia. Así, se detecta que factores que según la literatura científica son clave, como la motivación y la autoeficacia (Holton, 2005; Early, 2004), y oportunidades de aplicación al puesto de trabajo (Bartlett, 2001; Pidd, 2004; Burke \& Hutchins, 2008), están presentes en el contexto laboral de las personas que se han formado, y se dan con más intensidad en aquellas situaciones en que la transferencia es más elevada.

Estos resultados coinciden con los obtenidos mediante los métodos cualitativos aplicados en el estudio para evaluar la transferencia en cinco acciones formativas, que no se han presentado aquí al escaparse del objeto de este artículo: entrevistas a los superiores e informes del formador. A través de estas técnicas de recogida de información se pretendía obtener evidencias sobre la transferencia, más allá de la opinión de los participantes y se ha corroborado que las condiciones de trabajo unido al apoyo en el proceso de aplicación de los aprendizajes resultan claves para transferir. La información obtenida por estos agentes, aunque ha permitido corroborar resultados, ha resultado al mismo tiempo bastante homogénea en lo que se refiere a los posibles obstáculos en la transferencia pero, sin embargo, sí que ha permitido detectar en qué centros hay mayores dificultades para aplicar los aprendizajes. Esta información corrobora la alta desviación típica de algunos resultados entre los diferentes centros de trabajo donde se ha realizado la evaluación.

El colectivo médico se caracteriza por una alta autonomía en el ejercicio de sus funciones; a menudo, la prescripción médica la realiza en solitario, por lo que a veces el superior y los compañeros tienen poca información para valorar si ésta se ve afectada por la transferencia de la formación. Esto nos indica que en ciertos colectivos la información a partir de la percepción de los mismos participantes en la formación resulta clave para aproximarnos a la realidad del contexto de trabajo y las oportunidades de transferencia.

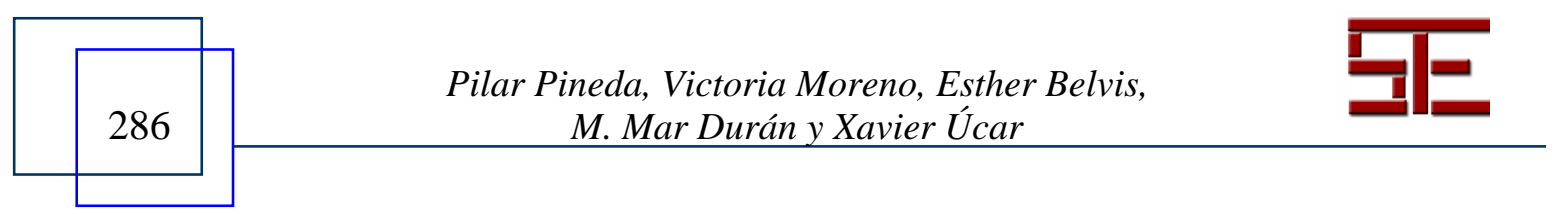




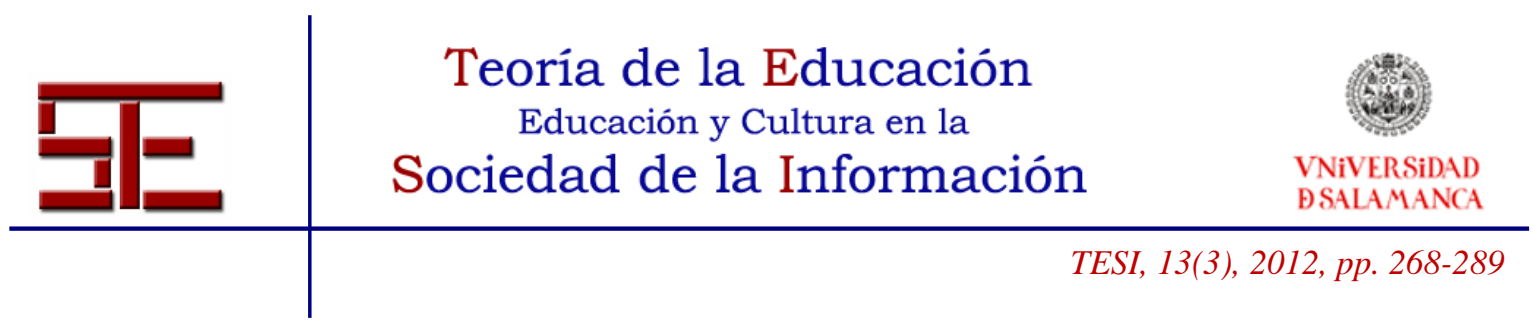

Esta coincidencia de resultados, unida a las características del colectivo, permite validar los datos cuantitativos y confirma que el cuestionario utilizado es una herramienta apropiada para evaluar la transferencia de la formación en el sector sanitario. En este sector, el cuestionario on line al participante es una herramienta útil, de fácil aplicación que puede ser utilizada en las organizaciones con los recursos de que disponen. Con esta herramienta podemos evaluar la permanencia de los aprendizajes, los cambios concretos en su actuación profesional que perduran en el tiempo y los factores que contribuyen a dichos cambios.

Sin embargo, para utilizar el sistema de evaluación creado en otros contextos, creemos necesario garantizar el acceso de los participantes a las nuevas tecnologías desde su puesto de trabajo para que puedan responder a la encuesta, y el apoyo de los órganos directivos a la evaluación, como una estrategia importante para dar apoyo a que todos los participantes completen la encuesta.

En el diseño de planes de evaluación vinculados a sectores específicos de trabajo se han de tener en cuenta el origen de la formación -quién es el responsable de que esta se lleve a cabo- y sus objetivos - los principales cambios que se pretenden introducir en los puestos de trabajo y las organizacione. Ambos aspectos son clave tanto para garantizar un mayor apoyo de la organización en el proceso de evaluación como un mayor conocimiento de las posibilidades de transferencia a los puestos de trabajo.

En el proceso de recogida de información es muy importante contar con el compromiso y colaboración de los centros y organizaciones ya que son estos los que pueden exigir y garantizar un mayor compromiso de sus trabajadores. Cuando la evaluación se realiza de forma externa y se contacta directamente con el participante, es muy posible que la recogida de información sea menor, o que esté sesgada por una mayor participación de los colectivos más motivados o de los centros que funcionan mejor. Este aspecto resulta capital, ya que las informaciones que obtenemos están supeditadas también a aspectos organizativos y de clima laboral. Sucede que los centros que necesitan analizar sus problemáticas en más profundidad para mejorar la transferencia e introducir cambios son precisamente aquellos de los que se obtiene menos información.

Concluimos que la evaluación con grandes muestras de formación y de centros de trabajo permite conocer a grandes rasgos cual es la situación de la formación en un sector y las principales problemáticas de la transferencia de la formación. Permite

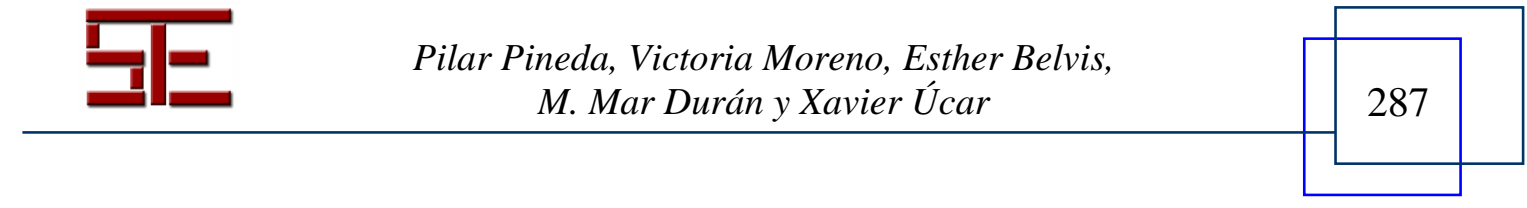




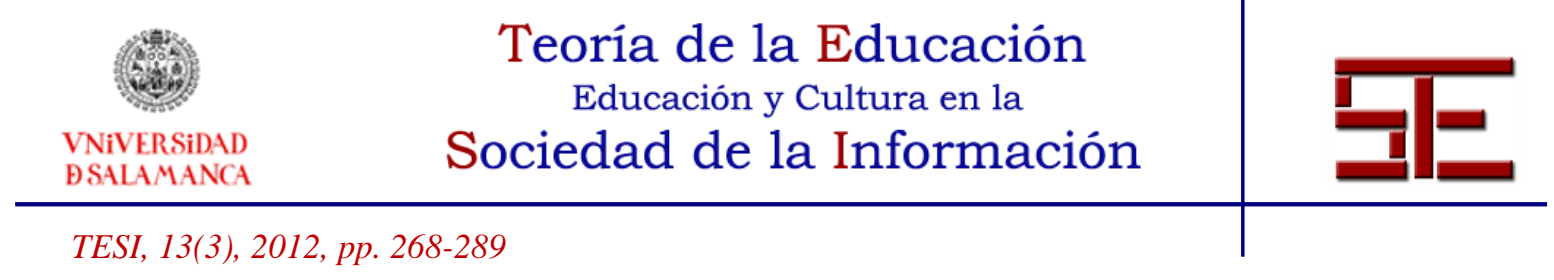

establecer a grandes rasgos los factores que pueden tener incidencia en la transferencia, sin entrar en la especificidad de cada uno de los centros de trabajo. Este tipo de estudios resulta útil para aquellos investigadores que de forma más específica quieran analizar la transferencia, ya que les permite tener un primer input de los aspectos que hay que tener en cuenta en el diseño y en la aplicación de los instrumentos.

\section{BIBLIOGRAFÍA.}

Baldwin, T. T; Ford, J. K. (1988). Transfer of training: a review and directions for future research. Personnel Psychology, 41, 63-105.

Bartlett., K. R. (2001). The Relationship between training and organizational Commitment: A Study in the health care field. Human Resource Development Quaterly, 12 (4), 335-352.

Burke, L. A y Hutchins; H. M. (2008). A Study of Best Practices in Training Transfer and Proposed Model of Transfer. Human Resource Development Quaterly, 19 (2), 107-125.

Holton, E.F. III., et al. (1996). The flawed four-level evaluation model. Human Resource Development Quarterly, 7, 5-21.

HOLTON, E. F. III. (2005). Holton's evaluation model: new evidence and construct elaborations. Advances in Developing Human Resources, 7 (37), 37-54.

Jhpiego. (2002). Transfer of learning: A guide for strengthening the performance of health care workers. North Carolina Univ. Chapel Hill School of Medicine, Jhpiego Corp.

Kirkpatrick, J. (2005). Transferring learning to behavior. $T+D, 59$ (4), 19.

Lim, D. H y Johnson, S. D. (2002). Trainee perceptions of factors that influence learning transfer. International Journal of Training and Development, 1, 36-48.

Nijman, D. J. M., Nijhof, W. J., Wognum, A. A. M., y Veldkamp, B. P. (2006). Exploring differential effects of supervisor support on transfer of training. Journal of European Industrial Training, 7, 529-549.

Noe, R. A. (1996). Trainees' attributes and attitudes: neglected influences on training effectiveness. Academy of Management Review, 11 (4) 736-749.

Pidd, K. (2004). The impact of workplace support and identity on training transfer. International Journal of Training and Development, 8 (4), 274-288.

Pineda, P. (2002). Gestión de la formación en las organizaciones. Barcelona: Ariel.

Pineda, P., Moreno, V., Úcar, X. y Belvis, E. (2007). Evaluación de la calidad de la formación continua en el sector de la educación infantil: informe ejecutivo. Barcelona.

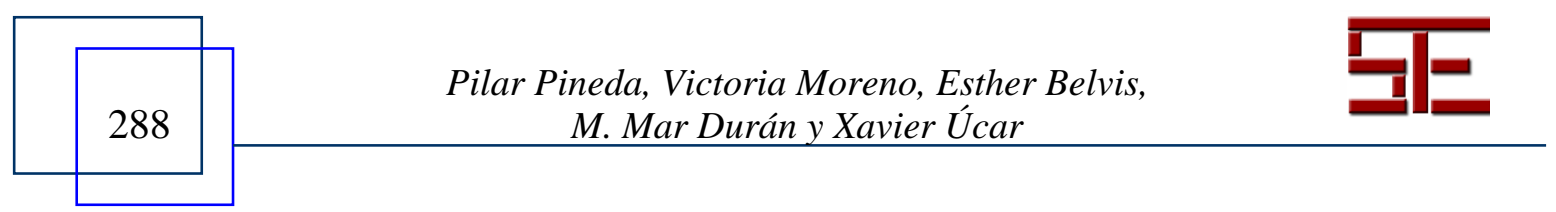




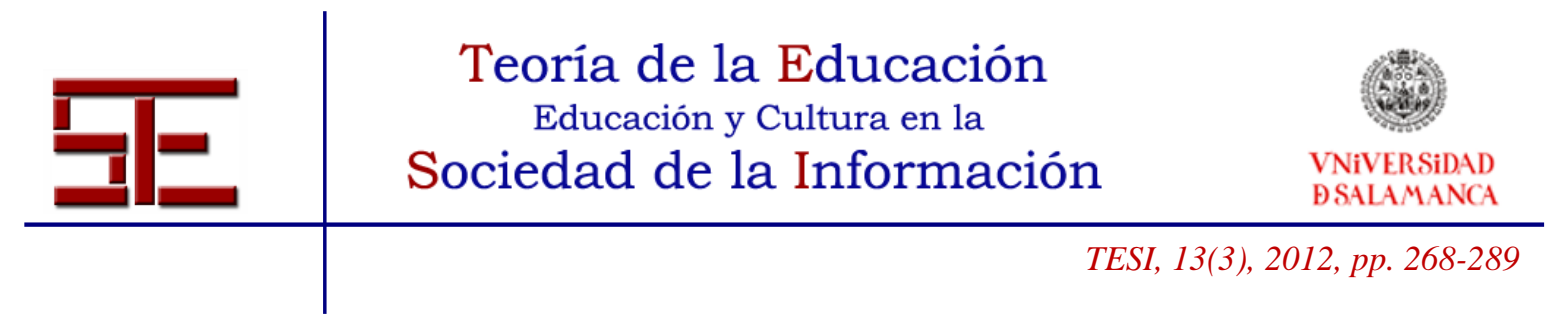

Pineda, P., Armengol, C., Belvis, E. \& Moreno, V. (2008). Avaluació de la formació en pràctica reflexiva: informe executiu. Barcelona.

Saks, A. y Belcourt, M. (2006). An investigation of training activities and transfer of training in organizations. Human Resource Management, 45 (4), 629-648.

Swanson, R. A. (1996). Evaluation systems in HRD. PLS evaluation system: Sales communication case study. Symposium Evaluation Systems in HRD, Minneapolis.

Thayer, P. W. \& Teachout, M. S. (1995). A climate for transfer model. Armstrong Laboratory/Human Resources, Brooks Air Force Base, TX.

UCF (2007). Pla de Formació en Us Racional de Medicament. Barcelona: UCF. Documento inédito.

Para citar el presente artículo puede utilizar la siguiente referencia:

Pineda Herrero, P., Belvis Pons, E., Duran-Belloch, M. M. y Úcar Martínez, X. (2012). Evaluación de la formación continua en el sector sanitario. Resultados de la formación en uso racional del medicamento. Revista Teoría de la Educación: Educación y Cultura en la Sociedad de la Información. 13(3), 268-289 [Fecha de consulta: dd/mm/aaaa]. http://campus.usal.es/ revistas_trabajo/index.php/revistatesi/article/view/9141/9374

Pilar Pineda, Victoria Moreno, Esther Belvis, M. Mar Durán y Xavier Úcar 